\title{
Calcium and Cyclic AMP Promote Axonal Regeneration in Caenorhabditis elegans and Require DLK-1 Kinase
}

\author{
Anindya Ghosh-Roy, ${ }^{1}$ Zilu Wu, ${ }^{1,2}$ Alexandr Goncharov, ${ }^{1,2}$ Yishi Jin, ${ }^{1,2}$ and Andrew D. Chisholm ${ }^{1}$ \\ ${ }^{1}$ Division of Biological Sciences, Section of Neurobiology and ${ }^{2}$ Howard Hughes Medical Institute, University of California San Diego, La Jolla, \\ California 92093
}

Axons of adult Caenorhabditis elegans neurons undergo robust regenerative growth after laser axotomy. Here we show that axotomy of PLM sensory neurons triggers axonal calcium waves whose amplitude correlates with the extent of regeneration. Genetic elevation of $\mathrm{Ca}^{2+}$ or cAMP accelerates formation of a growth cone from the injured axon. Elevated $\mathrm{Ca}^{2+}$ or cAMP also facilitates apparent fusion of axonal fragments and promotes branching to postsynaptic targets. Conversely, inhibition of voltage-gated calcium channels or calcium release from internal stores reduces regenerative growth. We identify the fusogen EFF-1 as critical for axon fragment fusion and the basic leucine zipper domain (bZip) protein CREB (cAMP response element-binding protein) as a key effector for branching. The effects of elevated $\mathrm{Ca}^{2+}$ or cAMP on regrowth require the MAPKKK (mitogen-activated protein kinase kinase kinase) DLK-1. Increased cAMP signaling can partly bypass the requirement for the bZip protein CEBP-1, a downstream factor of the DLK-1 kinase cascade. These findings reveal the relationship between $\mathrm{Ca}^{2+} /$ cAMP signaling and the DLK-1 MAPK (mitogen-activated protein kinase) cascade in regeneration.

\section{Introduction}

The ability of a neuron to undergo regenerative growth after injury is governed by the interplay between its intrinsic growth capacity and the local extracellular environment. In adult mammals, the peripheral nervous system is able to regenerate (Chen et al., 2007), whereas central (CNS) axons do not regrow efficiently. This distinction between central and peripheral axon regeneration reflects differences both in glial microenvironment (Yiu and $\mathrm{He}, 2006)$ and in intrinsic growth abilities (Filbin, 2003). However, the variables determining a neuron's intrinsic regrowth capacity remain poorly defined at a molecular level.

Calcium and cAMP signals have been implicated in axonal regeneration in many systems. Extensive studies of axotomy responses in cultured Aplysia neurons have shown that axonal injury results in a rapid increase of axonal $\mathrm{Ca}^{2+}$ (Ziv and Spira, 1995). This $\mathrm{Ca}^{2+}$ transient is thought to be attributable to a combination of $\mathrm{Ca}^{2+}$ diffusion through membrane breaches, depolarization-dependent influx via voltage-gated calcium channels (VGCCs) (Kulbatski et al., 2004), and $\mathrm{Ca}^{2+}$-induced $\mathrm{Ca}^{2+}$ release from internal stores (Ziv and Spira, 1993). Subsequent

\footnotetext{
Received Nov. 3, 2009; revised Dec. 11, 2009; accepted Dec. 23, 2009.

A.G. and Z.W. are associates and Y.J. is an investigator of the Howard Hughes Medical Institute (HHMI). This work was supported by National Institutes of Health (NIH) Grant R01 NS057317 (A.D.C.). We thank Mike Nonet (Washington University, St. Louis, M0) for js/s821, Ken Miller (Oklahoma Medical Research Foundation, Oklahoma City, OK) for pde-4 strains and reagents, Shohei Mitani (Tokyo Women's Medical University, Tokyo, Japan) for tm alleles, Benjamin Podbilewicz (Technion IIT, Haifa, Israel) for nsf-1(ty10), Cori Bargmann (Rockefeller University, New York, NY) and Loren Looger (Janelia Farm, HHMI) for G-CaMP clones, and Howard Baylis (Cambridge University, Cambridge, UK) for sponge constructs. Some strains were provided by the Caenorhabditis Genetics Center, which is supported by the NIH National Center for Research Resources. We thank members of our laboratories for discussions and Zhiping Wang for comments on this manuscript.

Correspondence should be addressed to Andrew D. Chisholm, Division of Biological Sciences, Section of Neurobiology, University of California San Diego, 9500 Gilman Drive, La Jolla, CA 92093. E-mail: chisholm@ucsd.edu.

DOI:10.1523/JNEUROSCI.5464-09.2010

Copyright $\odot 2010$ the authors $\quad 0270-6474 / 10 / 303175-09 \$ 15.00 / 0$
}

$\mathrm{Ca}^{2+}$-triggered activation of multiple signal transduction processes initiates repair. In vitro, the levels of calcium signaling can affect whether a severed axon can convert its stump into a new growth cone (Kamber et al., 2009).

One effect of increased $\mathrm{Ca}^{2+}$ is to activate $\mathrm{Ca}^{2+}$-dependent enzymes such as adenylate cyclase (AC). Axonal injury has been shown to cause an increase in axonal AC activity and cAMP levels for several hours (Appenzeller and Palmer, 1972; Carlsen, 1982). These observations raise the question of whether cAMP levels might be rate limiting in axonal regeneration. Some zebrafish spinal neurons with poor intrinsic regrowth capacity can be induced to regrow by exogenous cAMP (Bhatt et al., 2004). Exogenous dibutyryl-cAMP or stimulation of AC via forskolin also enhances regeneration in rat sciatic nerve (Pichichero et al., 1973) and of CNS axons in inhibitory microenvironments (Spencer and Filbin, 2004). Injection of cAMP mimics the growth-promoting effects of conditioning lesions (Neumann et al., 2002), suggesting cAMP levels are determinants of regrowth in dorsal root ganglion (DRG) neurons.

In other neurons, cAMP is not sufficient to enhance regrowth but can play a facilitating role. CAMP does not promote regeneration of axotomized retinal ganglion cells but can potentiate effects of growth factors on neuronal survival (Park et al., 2004). cAMP has a mild effect on regeneration of sensory neurons into spinal cord lesions ( $\mathrm{Lu}$ et al., 2004). It is unclear whether cAMP can promote axonal regeneration independent of modulating responsiveness to extrinsic factors, such as neurotrophins or myelin inhibitors, and whether it plays a permissive or rate-limiting role in vivo.

Caenorhabditis elegans neurons have a robust capacity to regrow after femtosecond laser surgery (Yanik et al., 2004; Wu et al., 2007), allowing the pathways regulating intrinsic regrowth capacity to be mapped out in detail. We show here that laser axo- 
tomy triggers axonal $\mathrm{Ca}^{2+}$ transients whose dynamics correlate with subsequent regrowth. We find that $\mathrm{Ca}^{2+} / \mathrm{cAMP}$ signals promote axonal regrowth, reconnection of axonal fragments, and formation of branches to the target region. These aspects of regenerative growth involve distinct effectors and are dependent on the DLK-1 pathway.

\section{Materials and Methods}

C. elegans genetics. Nematodes were raised on nematode growth medium agar plates between 15 and $25^{\circ} \mathrm{C}$ using standard procedures. We used the following mutants: egl-19(ad695gf, ad1006lf), unc-36(e251), unc-2(e55), itr-1(sa73), crt-1(bz29), unc-68(e540), pde-4(ce268, tm2536), gsa1(ce94gf), acy-1(md1756gf), kin-2(ce179), epac-1(ok655), eff-1(ok1021), aff-1(tm2214), nsf1(ty10), crh-1(tz2), jun-1(gk551), dlk-1(ju476), cebp-1(tm2807), atf-5(ok576), atf-6(ok551), atf$7(g k 715)$. All strains were confirmed by PCR genotyping or sequencing. We used the following transgenes: Pmec-7-GFP (muIs32), Pmec-4-GFP ( $z d I s 5)$, and Pmec-7-GFP::RAB-3 ( jsIs821) (Bounoutas et al., 2009).

Plasmid and transgene construction. For touch neuron-specific expression, we generated Gateway (Invitrogen) entry cDNA clones for the gene of interest by PCR and recombined them with a $\mathrm{P} m e c-4$ destination vector, pCZGY533, to generate the constructs detailed in supplemental Table 1 (available at www.jneurosci.org as supplemental material). For pan-neural expression of PDE-4, we used Prab-3-PDE-4 plasmid KG203 (Charlie et al., 2006). Transgenes were generated by standard methods with Pttx-3-RFP or -GFP as coinjection marker; total DNA concentration was $150 \mathrm{ng} / \mu \mathrm{l}$.

Laser axotomy and quantitative imaging. We performed laser axotomy essentially as described previously (Wu et al., 2007). Green fluorescent protein (GFP)- or G-CaMP-expressing animals were imaged using $491 \mathrm{~nm}$ laser excitation, a Yokogawa CSU-XA1 spinning-disk confocal head, and a Photometrics Cascade II EMCCD camera $(1024 \times 1024)$, controlled by $\mu$ Manager (www.micro-manager.org). For live imaging of G-CaMP fluorescence, we collected 114 ms exposures every $230 \mathrm{~ms}$ using the spinning-disk confocal head. Femtosecond laser power varied between 140 and $180 \mathrm{~mW}$ with a shutter opening time of 1.2-1.5 ms. We used MetaMorph (Molecular Devices) for fluorescence quantitation. Average fluorescence was measured in two equivalent regions of interest (ROIs), one centered on the PLM process and the other centered just outside the process. Net fluorescence $(F)$ in a given ROI was obtained by subtracting background fluorescence. Baseline fluorescence $\left(F_{0}\right)$ was obtained by averaging the fluorescence in five consecutive frames before axotomy. The change in fluorescence $\Delta F$ was expressed as the ratio of change with respect to the baseline $\left(F_{\mathrm{t}}-F_{0} / F_{0}\right)$. To follow $\Delta F / F_{0}$ over time at different distances from the axotomy site, we drew $2 \mu \mathrm{m}$ ROIs at intervals of 5 $\mu \mathrm{m}$. As G-CaMP intensity typically increased over a 10-12 $\mu \mathrm{m}$ axonal region within the first $230 \mathrm{~ms}$ of axotomy, we selected a $12-\mu \mathrm{m}$-long ROI to compare the initial $\Delta F / F_{0}$ between different conditions. As controls, we measured fluorescence in processes without performing axotomy or on axotomized GFP-labeled processes (supplemental Fig. $1 A-C$ and supplemental Video 2, available at www.jneurosci.org as supplemental material).

To quantify axon regrowth, we measured the total length of the PLM or ALM process (including all branches) from projections of confocal $z$-stacks. The distance from cut site to the point where the axon meets the soma was measured immediately after cutting and subtracted from the total length at the time of interest (typically 10 or $24 \mathrm{~h}$ after axotomy) to yield the length of regenerated processes. We also took into account the high rate of process fusion in some backgrounds: instances of axonal fusion were not included in the measures of average regrowth.

Statistical analysis. All statistical analyses used GraphPad Prism. Two-way comparisons used the Student's $t$ test, Mann-Whitney test, or Fisher's exact test for proportions. Comparisons in which the design involved more than two conditions used ANOVA and a Tukey's or Dunnett post test.

Drug experiments. BAPTA-AM, Nemadipine-A, forskolin, and H89 were obtained from Sigma and (with the exception of H89) were dissolved in DMSO before dilution in M9. H89 stocks were prepared in water before dilution in M9. We incubated worms in drug solution (containing Escherichia coli OP50) for $12 \mathrm{~h}$ before axotomy; control animals were incubated in M9-containing DMSO solvent to the equivalent concentration to that used in the specific experiment.

In the course of these drug experiments, we found that animals incubated in liquid consistently showed more PLM regrowth compared with 

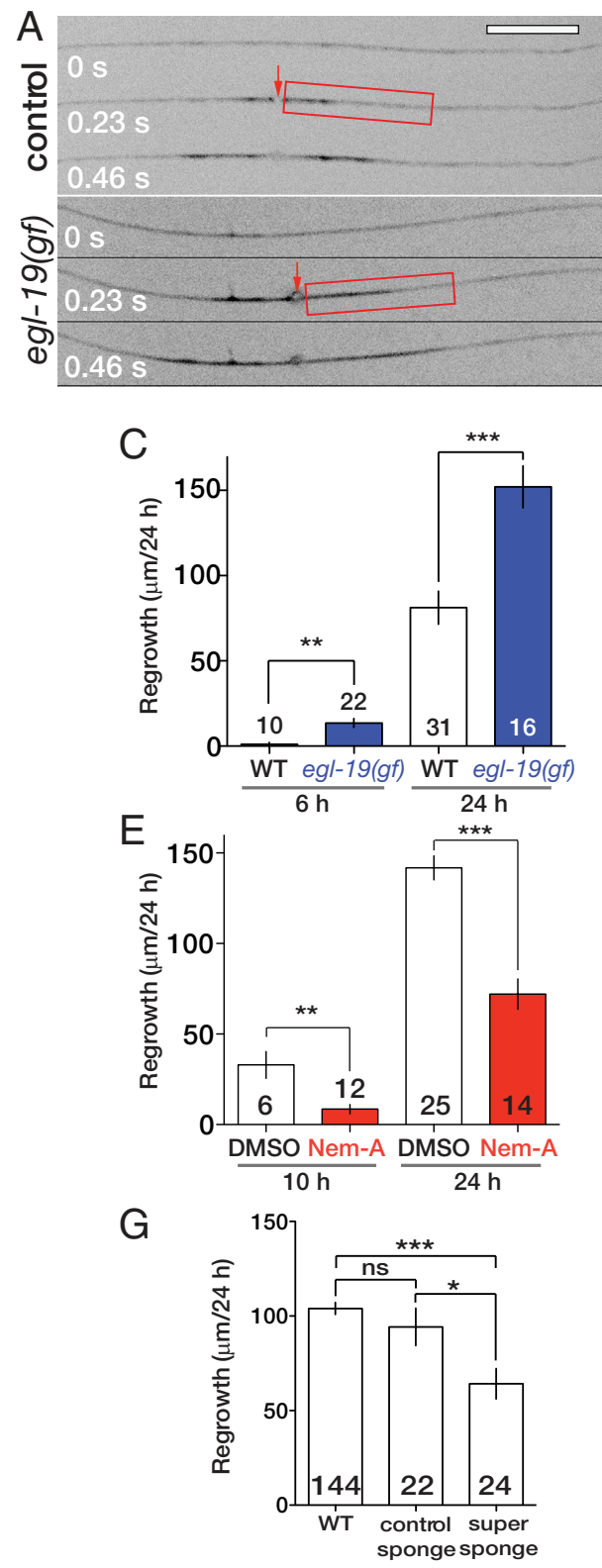

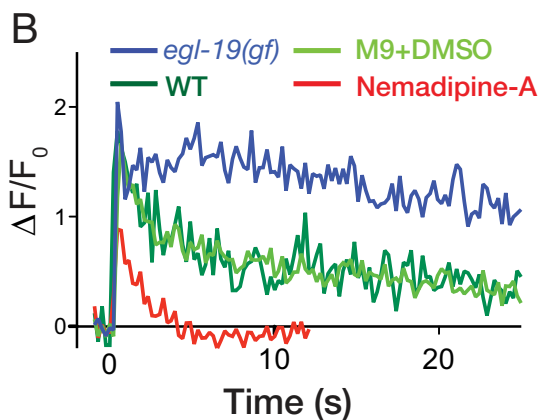

D

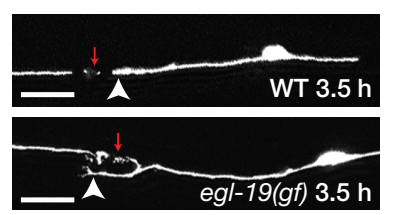

$\mathrm{F}$

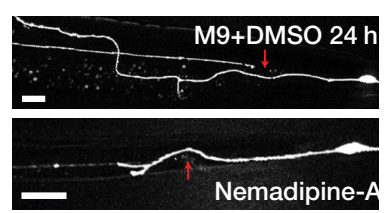

$\mathrm{H}$

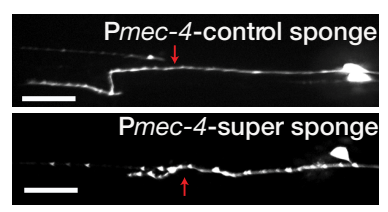

Figure 2. The L-type VGCC EGL-19 and internal stores contribute to the axotomy calcium transient and are required for regrowth. $\boldsymbol{A}$, Time frames from videos collected during axotomy performed on control and egl-19(ad695gf) worms expressing Pmec-4-G-CaMP 2.0 ( juEx2116). B, Effects of egl-19(ad695gf) (blue trace) and the EGL-19 inhibitor Nemadipine-A (red trace) on decay of the G-CaMP transient, measured close to the site of axotomy; representative traces are shown. C, egl-19(gf) mutants display increased axon regrowth 6 and $24 \mathrm{~h}$ after axotomy. $D$, At $3.5 \mathrm{~h}$, most wild-type animals do not show sprouting of the injured stump (arrowhead), whereas $\sim 50 \%$ of $\mathrm{egl}-19$ ( $\mathrm{g}$ ) animals display visible sprouting (arrowhead). Red arrows in $\boldsymbol{D}, \boldsymbol{F}$, and $\boldsymbol{H}$ indicate position of axotomy. $\boldsymbol{E}$, Nemadipine-A (Nem-A) reduces regrowth of PLM axons at 10 and $24 \mathrm{~h}$. F, PLM regrowth at $24 \mathrm{~h}$ in drug control conditions and after Nemadipine-A treatment. G, Touch neuron-specific expression of super sponge transgenes ( juEx2410) has a stronger inhibitory effect than do control sponge transgenes ( $j u E \times 2412$ );zdls5 background. $\boldsymbol{H}$, Representative PLM regrowth in control sponge- and super sponge-expressing animals. Numbers in bars indicate the number of axons from at least two independent experimental groups. Scale bars, $10 \mu \mathrm{m}$. Statistics: $t$ test; ${ }^{*} p<0.05 ;{ }^{* *} p<0.01 ;{ }^{* *} p<0.001$. WT, Wild type; ns, not significant.

animals growing on agar plates (supplemental Fig. $1 D$, available at www. jneurosci.org as supplemental material). Such differences may reflect the different physiology and growth rates of animals grown in liquid versus solid media, but their basis has not been explored in detail. All statistical comparisons were between groups grown under the same culture conditions and axotomized on the same day.

Electron microscopy. We cut the PLM axon in the L4 stage and imaged regrowth at 6 and $24 \mathrm{~h}$ after axotomy. Animals were fixed for electron microscopy at 24 or $36 \mathrm{~h}$, as described previously (Pujol et al., 2008).
Serial $50 \mathrm{~nm}$ sections were cut from the tail toward the anterior until the regrown axon had been completely sectioned.

\section{Results}

Laser axotomy of PLM neurons triggers a calcium transient that correlates with regenerative growth

After femtosecond laser axotomy of GFPlabeled PLM neurons in L4 stage $C$. elegans, the stump of the proximal axon forms a growth cone that then extends $\sim 100 \mu \mathrm{m}$ in $24 \mathrm{~h}$ (Wu et al., 2007). To analyze how laser axotomy might affect axonal calcium dynamics, we expressed the GFP-based calcium sensor G-CaMP 2.0 (Nakai et al., 2001; Akerboom et al., 2009) in touch neurons and imaged their fluorescence after laser axotomy using spinning-disk confocal microscopy. The posterior touch neurons (PLM) are a bilaterally symmetric pair of cells whose cell bodies lie in the lumbar ganglion and that extend a single anterior neurite of average length $300 \mu \mathrm{m}$ (in L4 larvae), with a collateral branch extending to the ventral nerve cord to form synapses. Laser axotomy of the PLM axon $\sim 50 \mu \mathrm{m}$ from the cell body (Fig. 1A) triggered a rapid local increase in G-CaMP intensity that propagated in a wave away from the cut site in both proximal and distal fragments of the axon (Fig. 1B; supplemental Video 1, available at www.jneurosci.org as supplemental material). Fluorescence of GFPlabeled processes was not affected by laser axotomy (supplemental Fig. S $1 a, b$ and supplemental Video 2, available at www. jneurosci.org as supplemental material). We measured the relative change in G-CaMP fluorescence $\left(\Delta F / F_{0}\right)$ as a function of time and distance from the site of injury (see Materials and Methods) and found that the amplitude and velocity of the G-CaMP transient decayed away from the cut site (Fig. 1C,D). Axotomy of PLM at other distances from the cell body triggered similar responses, as did laser surgery of ALM neurons (data not shown). We conclude that laser axotomy in vivo triggers an axonal calcium transient that can propagate along much of the cell.

The regrowth of G-CaMP-expressing PLM neurons was not significantly different from that of GFP-labeled axons (supplemental Fig. $1 C$, available at www. jneurosci.org as supplemental material) [Pmec-4-G-CaMP $j u E x 2116,124.1 \pm 49.7 \mu \mathrm{m}$ (mean $\pm \mathrm{SD}$ ) in $24 \mathrm{~h}$ vs Pmec-7-GFP muIs32, $90.4 \pm 30.9 \mu \mathrm{m} / 24 \mathrm{~h}$ ), indicating that the G-CaMP reporters did not interfere with regenerative growth. Regrowth of G-CaMP-labeled axons was variable, with SDs between one-half and one-third of the mean regrowth, as has also been observed for GFP-labeled C. elegans axons (Wu et al., 2007; Gabel et al., 2008). 
The extent of regrowth $10 \mathrm{~h}$ after axotomy was positively correlated with the amplitude of the initial transient (Fig. 1E) (Pearson $r=0.67 ; p=0.006$ ), suggesting that variation in outgrowth may result from differences in $\mathrm{Ca}^{2+}$ levels after axotomy. To address whether $\mathrm{Ca}^{2+}$ is required for PLM axon regrowth, we incubated G-CaMP transgenic animals in M9 buffer containing the membranepermeable $\mathrm{Ca}^{2+}$ chelator BAPTA-AM (Tsien, 1981). The $\Delta F / F_{0}$ triggered by axotomy in BAPTA was similar to that of control animals (supplemental Fig. $1 E$ and supplemental Video 3, available at www. jneurosci.org as supplemental material); however, baseline G-CaMP fluorescence was reduced $\sim 30 \%$ (arbitrary units) in the presence of BAPTA-AM ( $p<0.05$, Mann-Whitney test). Axon regrowth in animals incubated in BAPTA-AM was significantly reduced (Fig. $1 F$; supplemental Fig. $1 F$, available at www.jneurosci.org as supplemental material), suggesting that absolute levels of intracellular $\mathrm{Ca}^{2+}$ are determinants of regrowth.

\section{VGCCs and internal stores contribute} to axotomy-induced calcium transient and to regenerative growth

Increase in intracellular $\mathrm{Ca}^{2+}$ after axonal injury is thought to be caused by a combination of entry through the membrane breach, influx via VGCCs and release from internal stores. The VGCC $\alpha_{1}$ subunit EGL-19 is critical for $\mathrm{Ca}^{2+}$ transients in touch neurons (Suzuki et al., 2003). A gain-of-function mutation, egl-19(ad695gf), results in prolonged depolarization and extended calcium transients in touch neurons in response to touch stimuli or $\mathrm{KCl}$ (Lee et al., 1997; Kerr et al., 2000). We found that axotomy of PLM in egl-19(gf) produced a larger peak G-CaMP $\Delta F / F_{0}$ compared with wild type (Fig. $2 A$ ). The peak $\Delta F / F_{0}$ in egl-19(gf) animals was $1.5 \pm 0.08$ compared with $1.21 \pm 0.08$ in the wild type $(n>25$ per genotype; $p<0.01$, Mann-Whitney test). G-CaMP transients in egl-19(gf) decayed more slowly than in the wild type (Fig. $2 B$ ) and extended more broadly along the axon. Axonal regrowth at 6 and $24 \mathrm{~h}$ after axotomy was significantly increased in egl-19(gf) animals (Fig. $2 C)$. This increased growth in $e g l-19(g f)$ appears to be attributable to a combination of a reduced delay between axotomy and growth cone formation (Fig. 2D) and more rapid movement of the growth cone once formed. PLM axon length was slightly increased in egl-19(gf) mutants compared with $z d I s 5$ controls; however, other mutants described here do not affect touch neuron development (supplemental Fig. 2, available at www.jneurosci.org as supplemental material). In all experiments, $>90 \%$ of injured neurons survived and formed new growth cones from the stump of the severed axon. Thus, elevated calcium can promote regrowth independent of effects on neuronal survival.

egl-19 is essential for embryonic development (Lee et al., 1997), and a viable partial loss of egl-19 function mutation did not significantly affect G-CaMP responses or regeneration (sup- plemental Fig. $3 A, B$, available at www.jneurosci.org as supplemental material). We therefore used a specific antagonist of EGL-19, Nemadipine-A (Kwok et al., 2006), to inhibit VGCC function in adult animals. Nemadipine-A treatment did not affect baseline G-CaMP fluorescence (supplemental Fig. 3C, available at www. jneurosci.org as supplemental material) but significantly reduced $\Delta F / F_{0}$ after axotomy (Nemadipine-A, $0.95 \pm 0.07$ vs M9 plus DMSO, $1.37 \pm 0.1 ; n>16$ per condition; $p<0.01, t$ test $)$. Nemadipine-A significantly reduced axonal regrowth at 10 and $24 \mathrm{~h}$ (Fig. 2 E, F), indicating that EGL-19-mediated $\mathrm{Ca}^{2+}$ influx is important for axonal regrowth. In contrast, animals lacking the UNC-36 $\alpha_{2} / \delta$ accessory channel subunit or the non-L-type VGCC $\alpha_{1}$ subunit UNC-2 did not display significantly reduced regrowth (supplemental Fig. 3D, available at www.jneurosci.org as supplemental material), suggesting EGL-19 is responsible for most of the $\mathrm{Ca}^{2+}$ influx in the axotomy-triggered transient.

We next tested the role of internal stores in axotomy-triggered calcium dynamics and regrowth. The inositol trisphosphate $\left(\mathrm{IP}_{3}\right)$ receptor is critical for calcium-induced calcium release from internal stores (Berridge, 1993). Complete loss of function in the $C$. elegans $\mathrm{IP}_{3}$ receptor gene itr-1 retards animal development (Dal Santo et al., 1999), and itr-1 partial loss-of-function adults displayed normal PLM regrowth (supplemental Fig. $3 D$, available at 

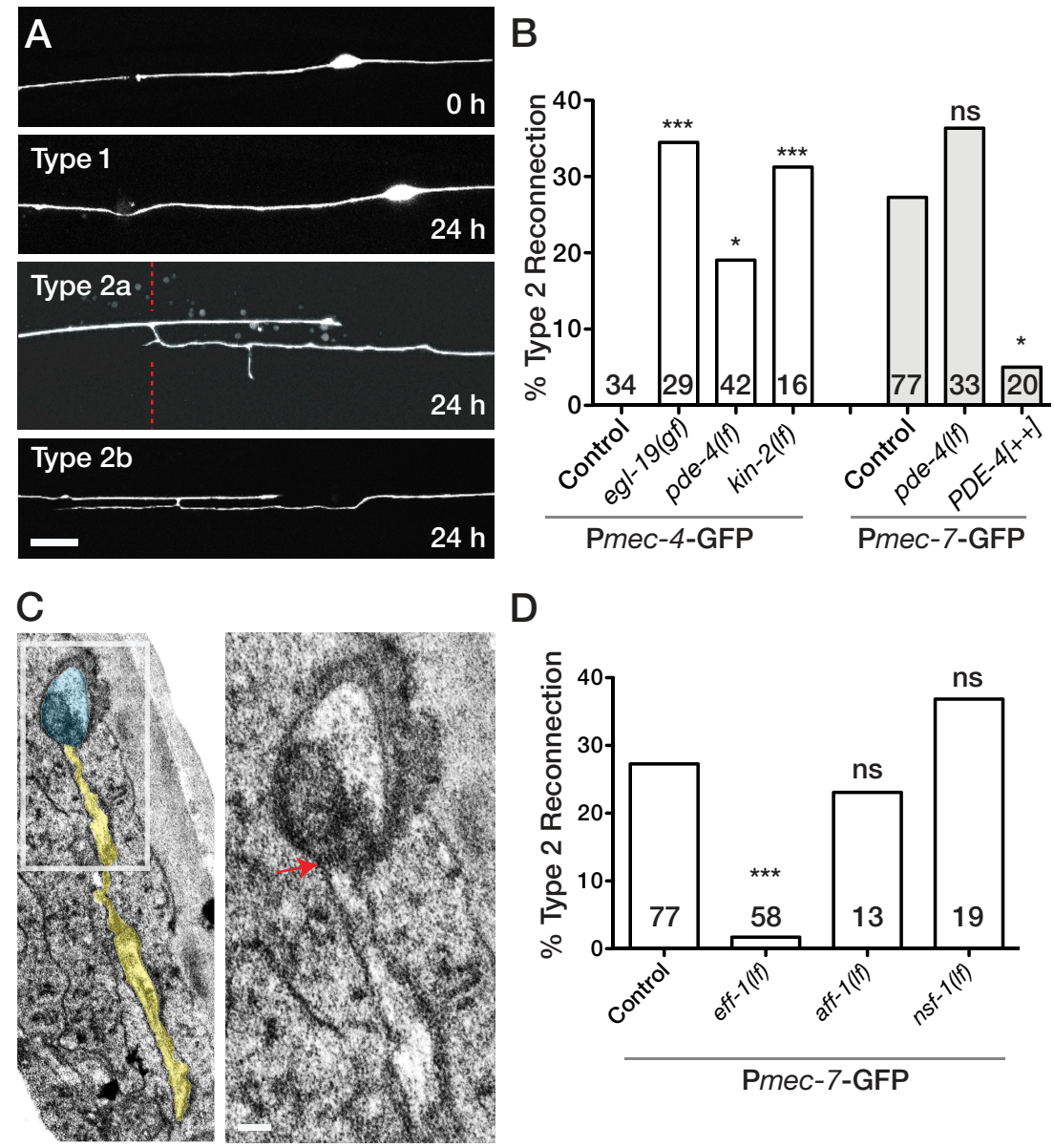

\section{D}

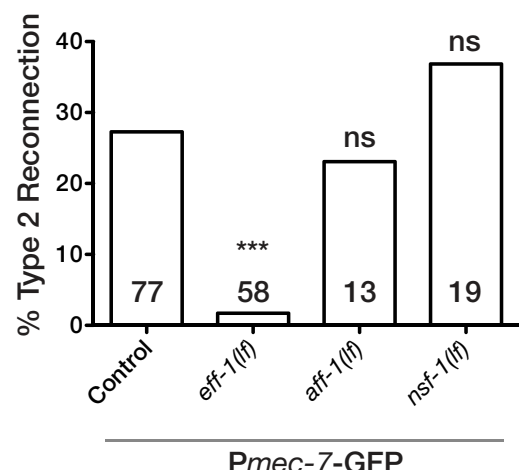

Figure 4. Reconnection of axotomized axon fragments is promoted by $\mathrm{Ca}^{2+}$ and cAMP and involves EFF-1. $\boldsymbol{A}$, Types of reconnection or contact observed between regrowing axons and distal fragments (examples from $p d e-4$ (If) zdls 5 background; confocal projections). Type 1 contact, Proximal and distal stumps reconnect without formation of a growth cone; Type 2 contact, the proximal stump forms a new growth cone that extends before turning (2a) or branching ( $2 b)$ to fuse collaterally with the distal fragment. The dotted red line indicates the region of an EM section in C. Scale bar, $10 \mu \mathrm{m}$. B , Type 2 reconnection occurs in $<5 \%$ of $z d l s 5$ control animals and is elevated in egl-19(gf), pde-4(If), and kin-2(If) mutants (Fisher's exact test; number of axons are shown in bars). Reconnection occurs in $\sim 25 \%$ of control muls32 animals, is slightly elevated in pde-4(If), and is significantly inhibited by touch neuron-specific expression of PDE-4 (Pmec-4-PDE-4). C, Electron micrographs of the junction of the distal fragment (blue shading) and regrowing axon (yellow) from the animal shown in $A$; the inset shows continuity of cell membranes at fusion (arrow). Scale bar, $100 \mathrm{~nm}$ (inset). D. Type 2 contacts in muls 32 background are reduced in animals lacking eff- 1 and are unaffected by loss of function in aff- 1 or $n s f-1 .{ }^{*} p<0.05,{ }^{* * *} p<0.01$, Fisher's exact test. ns, Not significant.

tion of endogenous $\mathrm{IP}_{3}$. As low-affinity $\mathrm{IP}_{3}$ sponge transgenes have some effect on regrowth, these constructs may interfere with additional roles of the ITR-1 N-terminal domain.

These results indicate that calcium release from internal stores is involved in the axotomy-induced $\mathrm{Ca}^{2+}$ transient and that this release is important for regenerative growth. The endoplasmic reticulum chaperone calreticulin (CRT-1) and the ryanodine receptor UNC-68 promote $\mathrm{Ca}^{2+}$ release from internal stores in necrotic cell death of touch neurons ( $\mathrm{Xu}$ et al., 2001). However, crt-1 or unc-68 null mutants displayed normal axonal regrowth (supplemental Fig. 3D, available at www. jneurosci.org as supplemental material), suggesting the mechanisms of axotomytriggered calcium release differ from those involved in necrosis.

\section{Genetic elevation of cAMP and} protein kinase A mimics the effects of elevated $\mathrm{Ca}^{2+}$

$\mathrm{Ca}^{2+}$ stimulates production of cAMP by activating $\mathrm{Ca}^{2+}$-sensitive AC. cAMP is subsequently degraded to 5'-AMP by phosphodiesterases. To test whether neuronal cAMP is rate limiting for regrowth in C. elegans axons, we tested a gain-offunction mutation in the neuronal AC $a c y-1$ and loss-of-function mutations in the neuronal phosphodiesterase pde-4 (Charlie et al., 2006). We also tested an activating mutation in the neuronal $\mathrm{G} \alpha_{\mathrm{S}}$ subunit $g s a-1$, which acts upstream of $a c y-1$ (Schade et al., 2005). gsa-1(gf), acy$1(g)$, and pde-4(lf) mutants all displayed increased regrowth $24 \mathrm{~h}$ after axotomy, comparable to egl-19(gf) (Fig. $3 A-C$ ). PLM regrowth was not further enhanced in pde-4(lf) egl-19(gf) double mutants, consistent with $\mathrm{Ca}^{2+}$ and cAMP acting in

www.jneurosci.org as supplemental material). We therefore attempted to interfere with ITR-1 function specifically in touch neurons using a dominant-negative approach. Overexpression of the N-terminal $\mathrm{IP}_{3}$-binding domain of ITR-1 can cellautonomously inhibit ITR-1 function; such "IP 3 sponges" likely act, in part, by titrating endogenous $\mathrm{IP}_{3}$ (Walker et al., 2002). We expressed either the wild-type ITR- $1 \mathrm{IP}_{3}$-binding domain, or low-affinity ("control sponge") and high-affinity ("super sponge") mutant forms of the domain, in touch neurons. These $\mathrm{IP}_{3}$ sponge constructs did not affect development of the touch neurons but significantly reduced the G-CaMP transient after axotomy (super sponge transgenes, $0.75 \pm 0.9$ vs Pmec-4-GCaMP alone, $1.16 \pm 0.1 ; n>15$ per condition; $p<0.01$, MannWhitney test). Presence of sponge transgenes strongly reduced regrowth (Fig. 2G,H). The super sponge (high $\mathrm{IP}_{3}$ affinity) caused the strongest reduction in regrowth; low-affinity $\mathrm{IP}_{3}$ sponge transgenes had a smaller effects on regrowth. The different effects of the low- and high- affinity $\mathrm{IP}_{3}$ sponges suggest that some growth-inhibiting effects of these constructs reflect titra- a common pathway to promote regrowth. Treatment with the AC activator forskolin caused a small increase in total regrowth that was not statistically significant (data not shown). However, forskolin treatment significantly overcame the block to regeneration caused by ITR-1 super sponge expression (Fig. 3D); after incubation in $100 \mu \mathrm{M}$ forskolin, super sponge-expressing PLM axons regrew $84.6 \pm 11.5 \mu \mathrm{m} / 24 \mathrm{~h}$ (mean $\pm \mathrm{SEM}$ ) compared with super sponge-expressing axons incubated in M9 buffer $(54.4 \pm 6.9 \mu \mathrm{m} / 24 \mathrm{~h})(p<0.05, t$ test $)$. Conversely, $p d e-4 \mathrm{mu}-$ tants displayed normal $\mathrm{Ca}^{2+}$ dynamics after axotomy (data not shown). These results are consistent with cAMP acting downstream or in parallel to $\mathrm{Ca}^{2+}$ in regrowth.

We focused on pde-4 mutants to further dissect the effects of elevated cAMP on regeneration. In the wild type ( $z$ dIs5), under our conditions of axotomy, the new growth cone formed by PLM does not begin to extend until 2-6 h after cutting. At $7 \mathrm{~h}$ after axotomy, wild-type axons had regrown $10.5 \pm 2.5 \mu \mathrm{m}(n=11)$, whereas pde-4(ce268) mutants displayed $21.2 \pm 2.7 \mu \mathrm{m}$ regrowth ( $n=19 ; p<0.001, t$ test). These findings suggest that elevation of 
cAMP promotes a more rapid initiation of growth cone formation, and subsequently more rapid axonal elongation than in the wild type. We next wanted to address whether lowering cAMP levels would impede regrowth. To titrate endogenous cAMP, we overexpressed PDE-4 in touch neurons and found that this significantly reduced axonal regeneration (Fig. 3C). Together, these results suggest that cAMP is both necessary and can be rate limiting for PLM regenerative growth.

Protein kinase A (PKA) and the CAMPGEF Epac are major cytosolic effectors of cAMP signals. To test whether PKA can promote regeneration, we examined mutants lacking the PKA regulatory subunit KIN-2 [kin-2(ce179)], which display constitutive activation of PKA (Schade et al., 2005). The effects of kin-2(ce179) on PLM regeneration closely resembled those of pde-4(lf), suggesting PKA might mediate the effects of cAMP on regrowth (Fig. 3C). As complete loss of function in the PKA catalytic subunit kin-1 results in lethality, we used the PKA inhibitor H89 to reduce PKA activity and found that this treatment reduced regrowth in a dose-dependent manner (Fig. 3E,F). C. elegans has a single cAMP-GEF, epac-1. epac-1 null mutants displayed normal regrowth after axotomy, and the enhanced regrowth of $p d e-4$ mutants was not suppressed in pde-4 epac- 1 double mutants (Fig. 3C). We conclude that PKA is the major effector of cAMP in PLM regrowth.

\section{Reconnection of the regrowing axon} with the distal fragment is enhanced by $\mathrm{Ca}^{2+} / \mathrm{cAMP}$ signaling and involves the fusogen EFF-1

After axotomy, the proximal and distal axon fragments occasionally reconnect by apparent fusion, as judged by apposition of GFP-labeled fragments and the failure of the distal fragment to subsequently degenerate. In some cases, the ends of the two axon fragments reconnected without extensive growth, which we term "type 1" connection. Most frequently, we observed "end-to-side" or "type 2" reconnection, in which the regrowing process formed a growth cone that extended before connecting to the side of the distal fragment (Fig. $4 A$ ). In most cases, the regrowing axon terminated when it met the distal fragment (type 2a), but, occasionally, it extended a collateral branch that appeared to be fused with the distal fragment (type 2b). In a small number of cases, the regrowing axon fasciculated with the distal fragment, although no clear reconnection could be seen.

The frequency of axonal reconnections in C. elegans depends on the transgenic background and method of axotomy (Bourgeois and

B

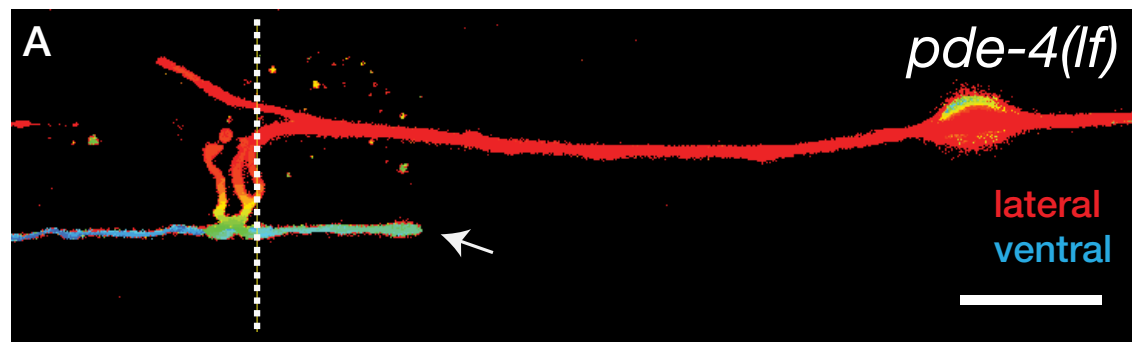

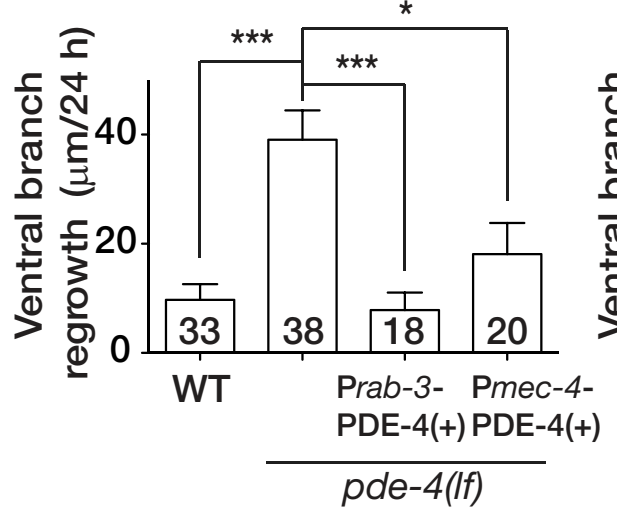
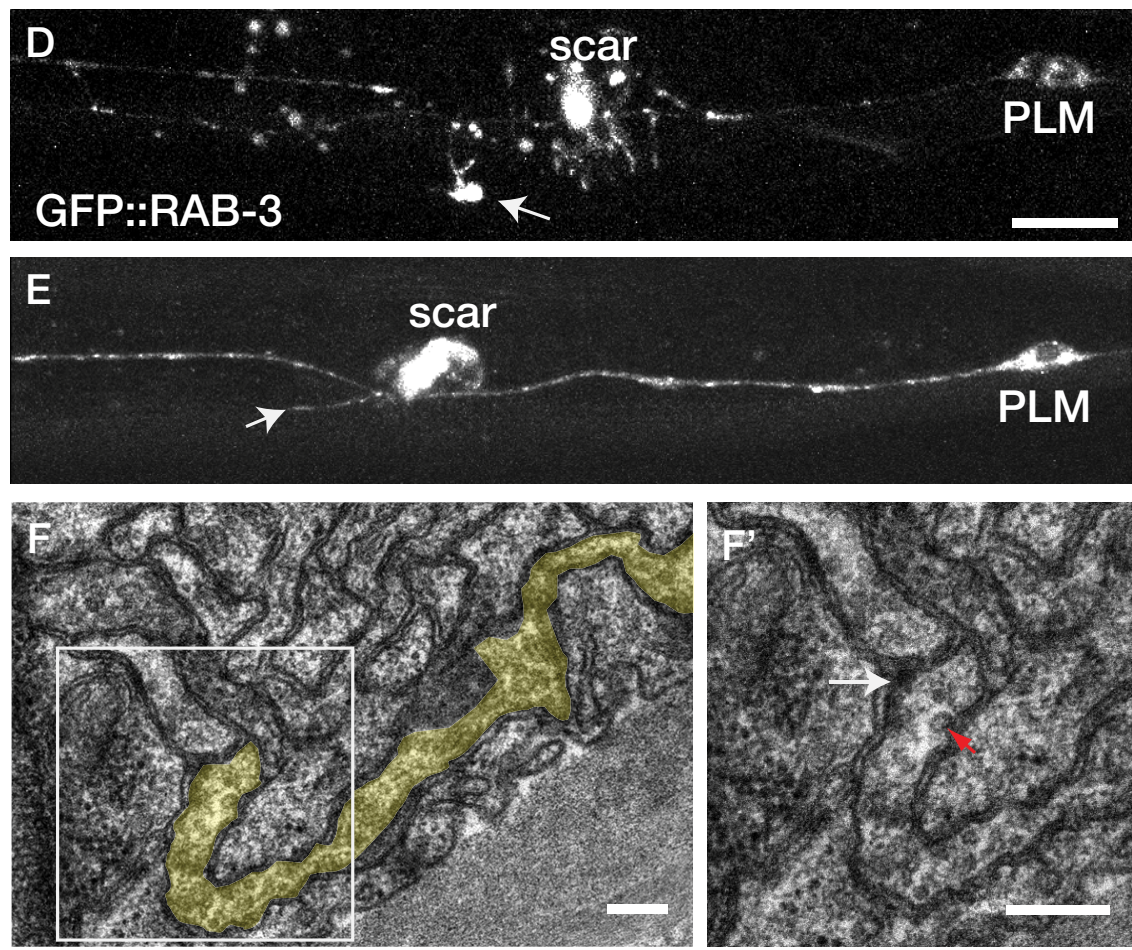

Figure 5. CAMP promotes regrowth of ventral synaptic branches in PLM. $\boldsymbol{A}$, Regrowing axons in pde-4(If) frequently extend ventrally directed branches (arrow) thatreachtheventral cord; the depth-coded projection of confocalz-stackisshown. Surfacefocal planes are red, and deep planes are blue. The dotted line indicates the position of the section shown in $\boldsymbol{F}$ and $\boldsymbol{F}^{\prime}$. $\boldsymbol{B}$, The total length of ventral branches is increased in $p d e-4$ mutants; this effect is rescued by expression of PDE-4 under the control of the rab-3 or mec-4 promoters. C, Incubation in forskolin promotes PLMventral branching in wild-type (muls32) animals; both 100 and $500 \mu \mathrm{m}$ forskolin promote ventral branching, although this is significant only for $500 \mu \mathrm{M}$ (Kruskal-Wallis test). D,E, GFP::RAB-3 ( $j$ s/s821) is enriched at the tips of regrowing branches that reach the ventral cord ( $\boldsymbol{D}$, arrow) and not in branches that extend laterally $(\boldsymbol{E}$, arrow); the pde-4(If) background is shown. Autofluorescent scars from laser surgery are indicated. $\boldsymbol{F}$, Electron micrograph of a regrown PLM branch (shaded yellow). $\boldsymbol{F}^{\prime}$, enlargement of the presynaptic area. White arrow, Electron dense zone; red arrow, $\sim 35$-nm-diameter synaptic vesicle-like structure. Scale bars; $\boldsymbol{A}, \boldsymbol{D}, \boldsymbol{E}, 10 \mu \mathrm{m} ; \boldsymbol{F}, \boldsymbol{F}^{\prime}, 100 \mathrm{~nm}$. Statistics:ttest; ${ }^{*} p<0.05 ;{ }^{* * *} p<0.001$.
Ben-Yakar, 2008; Guo et al., 2008). Under our axotomy conditions, using the transgene $z d I s 5, \sim 5 \%$ of severed PLM axon fragments reconnect, whereas in other transgenic backgrounds such as $m u I s 32, \sim 25 \%$ of PLM axons reconnect (Fig. $4 B$ ). We took 
A
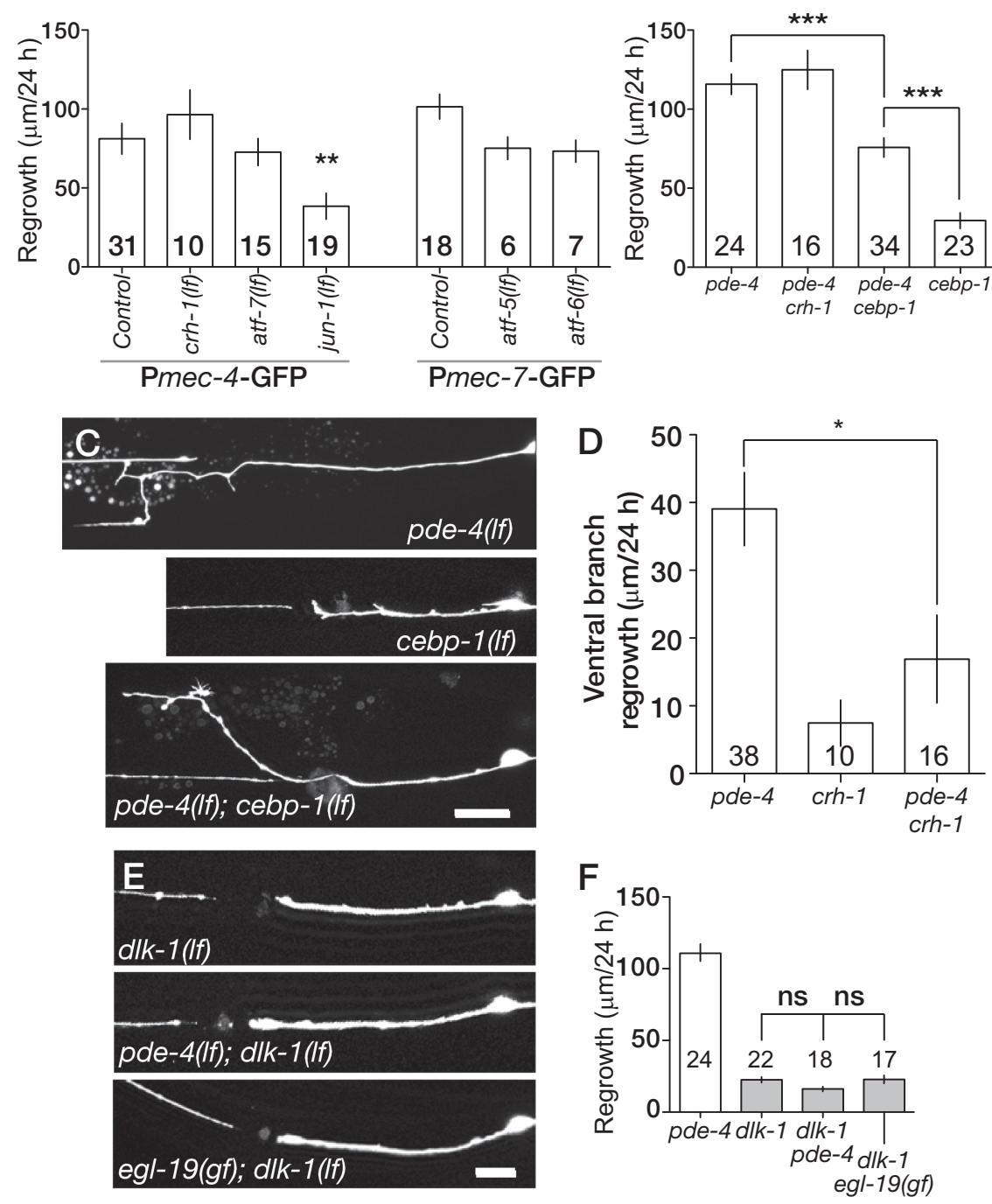

Figure 6. Effects of elevated CAMP require DLK-1 kinase and are partly dependent on the bZip protein CEBP-1. A, PLM regrowth in mutants lacking bZip transcription factors crh-1 (CREB), atf-7 (ATF7), jun-1 (c-Jun), atf-5 (ATF5), and atf-6 (ATF6). Regrowth is reduced in jun-1 mutants but not in other bZip mutants. $\boldsymbol{B}, \boldsymbol{C}$, Quantitation $(\boldsymbol{B})$ and confocal images $(\boldsymbol{C})$ of PLM regrowth in pde-4(If), cebp-1(If), and pde-4(If) cebp-1(If) double mutants. $\boldsymbol{D}$, crh-1 suppresses the enhanced ventral branching of $p d e-4$ (If). $\boldsymbol{E}, \boldsymbol{F}$, Confocal images $(\boldsymbol{E})$ and quantitation $(\boldsymbol{F})$ of PLM regrowth in dlk-1(ju476lf) and double mutants. Statistics: $t$ test; ${ }^{*} p<0.05$; ${ }^{* *} p<0.01$; ${ }^{* * *} p<0.001$. ns, Not significant.

advantage of the low rate of reconnection in the $z d I s 5$ background to test whether elevated $\mathrm{Ca}^{2+}$ or cAMP or activated PKA could increase fusion rates. We found that egl-19(gf), pde-4(lf), and kin-2(lf) mutants all displayed significantly elevated rates of fusion (Fig. $4 A, B$ ). pde-4 mutants displayed slightly elevated rates of reconnection in the muIs32 background, whereas touch neuron-specific overexpression of PDE-4 strongly inhibited fusion (Fig. $4 B$ ), suggesting that elevated cAMP can act cell autonomously to promote reconnection.

To determine whether such reconnections involved physical fusion of cell membranes, we performed serial section electron microscopy of a type 2 a reconnection in a pde- 4 mutant (Fig. $4 A$ ). We found that the plasma membrane of the regrown PLM axon (Fig. 4C, yellow shading) was continuous with that of the distal fragment (Fig. $4 C$, blue), confirming that the two cell fragments had fused. To determine the cellular components involved in fusion, we tested candidate fusogens. The membrane protein EFF-1 promotes homotypic fusion of epithelial and muscle cells
(Mohler et al., 2002). We found that type 1 and type 2 reconnection was almost eliminated in eff-1 null mutants compared with controls (Fig. 4D); instead, the regrowing axon branched extensively or fasciculated with the distal fragment (supplemental Fig. $4 A, B$, available at www. jneurosci.org as supplemental material). Overall regrowth was reduced but not eliminated in eff-1 mutants (supplemental Fig. 4C, available at www.jneurosci.org as supplemental material), indicating that the regrowing axons are capable of contacting the distal fragment but are specifically unable to fuse.

\section{Elevated cAMP promotes regrowth of} ventrally directed synaptic branches In normal development, PLM extends a single ventral branch that forms synapses with interneurons in the ventral cord (Fig. $1 A)$. In the wild type, PLM only occasionally regrew a branch toward the ventral cord after axotomy, whereas in pde-4 mutants, the regrowing PLM processes extended or branched to the ventral cord at a significantly higher frequency (compare Figs. $5 A, B, 3 A, B)$. Transgenic rescue experiments indicated that this effect of pde-4 on ventral outgrowth was cell autonomous (Fig. 5B). Treatment with forskolin also significantly increased the ability of regrowing PLM neurons to form ventral branches (Fig. 5C). To assess whether such regrown ventral branches formed presynaptic specializations, we first examined a presynaptic marker, GFP::RAB-3, which is concentrated at the synaptic branch in unoperated PLM neurons (Bounoutas et al., 2009). In regrowing pde-4 mutant PLM axons, GFP::RAB-3 became enriched at the tips of new branches if they reached the ventral cord, but not if they remained lateral (Fig. 5D,E). Using electron microscopy, we reconstructed a regrown ventral branch in a pde- 4 mutant and found that the branch tip contained multiple synaptic vesicles and a presynaptic density-like structure apposed to a neuronal process (Fig. $5 F, F^{\prime}$ ). These observations suggest that in pde-4 mutants, regrowing PLM ventral branches can reform synaptic connections to ventral cord neurons.

cAMP-enhanced regeneration requires the DLK-1 kinase and involves its target basic leucine zipper domain protein CEBP-1 Some effects of elevated $\mathrm{Ca}^{2+}$ or cAMP on axon regeneration may be mediated at the transcriptional level (Cai et al., 2002). In neuronal development and physiology, $\mathrm{Ca}^{2+}$ and cAMP often act via basic leucine zipper domain (bZip) factors such as cAMP response element-binding protein (CREB) (Sands and Palmer, 2008). Moreover, several bZip genes are upregulated after axonal injury in vertebrates, including c-Jun and ATF3 (Tsujino et al., 2000); c-Jun is partly required for regeneration of the facial nerve (Raivich et al., 2004). We therefore tested a set of homologous $C$. elegans bZip genes for requirements in axonal regeneration. jun-1 
mutants displayed significantly reduced regrowth, implying a conserved requirement for c-Jun in regeneration (Fig. 6A). In contrast, null mutations in crh-1 (CREB) or in several other bZip genes did not affect PLM regrowth (Fig. 6A). We constructed double mutants between pde-4 and null mutations in several bZip genes. Loss of function in crh-1 did not affect the overall increased regrowth of pde-4 mutants (Fig. $6 \mathrm{~B}$ ). However, crh$1(\mathrm{lf})$ significantly suppressed the formation of ventral branches caused by pde-4(lf) (Fig. 6D), suggesting that CRH-1 might mediate the effects of cAMP on ventral branching.

We recently showed that the bZip protein CEBP-1, which is a member of the CCAAT/Enhancer-binding protein subfamily, is required for C. elegans axon regrowth (Yan et al., 2009). CEBP-1 acts downstream of the DLK-1 MAP (mitogen-activated protein) kinase cascade, which is essential for C. elegans motor and touch axon regeneration (Hammarlund et al., 2009; Yan et al., 2009). Loss of $p d e-4$ function significantly suppressed the regeneration block in cebp-1(tm2807), such that $p d e-4$ cebp-1 double mutants displayed a level of regrowth intermediate between either single mutant (Fig. $6 B, C)$. In contrast, $d l k-1$ double mutants with $p d e-4$ or egl-19(gf) were indistinguishable from $d l k-1$ single mutants in their regrowth kinetics (Fig. 6E,F). Axotomy-triggered G-CaMP transients appeared normal in $d l k-1$ mutants (data not shown), consistent with DLK-1 acting downstream or in parallel to $\mathrm{Ca}^{2+}$ and cAMP signals. These findings suggest that cAMP elevation cannot bypass the requirement for DLK-1, but that increased cAMP can promote activity of other DLK-1 targets besides CEBP-1.

\section{Discussion}

C. elegans has recently emerged as a tractable model for studies of regenerative responses in neurons. Using imaging, genetics, and pharmacology, we have shown that C. elegans neurons respond to axotomy with a calcium transient whose magnitude is a critical determinant of the ability of the damaged neuron to regrow. We find that $\mathrm{Ca}^{2+}$, cAMP, and PKA are both required and rate limiting for normal PLM axon regrowth. Increased $\mathrm{Ca}^{2+}$ or CAMP signaling has strikingly similar effects on the PLM axonal response to injury, including accelerated formation of motile growth cones by the injured proximal stump, more rapid extension of growth cones once formed, increased formation of synaptic branches to the correct target area, and enhanced reconnection between the damaged axon fragments. Overall, our data are consistent with $\mathrm{Ca}^{2+}$, cAMP, and PKA acting in a single pathway, although we cannot exclude that $\mathrm{Ca}^{2+}$ and cAMP may also have functions independent of each other.

We found that both the VGCC EGL-19 and the $\mathrm{IP}_{3}$ receptor ITR-1 contribute to the local calcium transient caused by laser axotomy and to subsequent regrowth. These results imply that both EGL-19 and ITR-1 are present in axons and are activated in response to laser damage. The subcellular localization of EGL-19 in neurons has not yet been reported; in other organisms, there are few examples of axonally localized VGCCs. However, stretch injury of mammalian axons can trigger axonal calcium influx via VGCCs (Wolf et al., 2001). Stretch injury is thought to activate mechanosensitive $\mathrm{Na}^{+}$channels, causing membrane depolarization and opening of VGCCs. By analogy, we speculate that membrane damage caused by laser axotomy locally depolarizes the axon and triggers opening of VGCCs in the PLM axon. The $\mathrm{Ca}^{2+}$ influx across the membrane could then trigger calcium-induced calcium release from internal membrane stores in axons. AntiITR-1 immunostaining is present in the C. elegans nerve ring, consistent with axonal localization (Baylis et al., 1999). As C. elegans neurites appear to contain endoplasmic reticulum (Rolls et al., 2002), ITR-1 is likely able to act locally to control calcium dynamics after damage.

Elevation of $\mathrm{Ca}^{2+}$ and cAMP promotes reconnection by fusion of the regrowing axon and its severed distal segment. Fusion of severed axonal fragments was first inferred from electrophysiological observations in crayfish (Hoy et al., 1967) and has been observed in annelid and mammalian axons (Lore et al., 1999). In C. elegans, the rate of reconnection is sensitive to several variables, including the mode of laser cutting, the method of immobilization, and the transgenic background (Bourgeois and Ben-Yakar, 2008; Guo et al., 2008). The ability of the two fragments to fuse implies that the damaged distal fragment maintains a level of responsiveness to its environment. This possibility is consistent with recent findings that axotomy stimulates translation in the distal fragments of injured C. elegans axons (Yan et al., 2009), suggesting mechanisms by which the distal fragment could actively participate in the fusion process. Furthermore, our observation that reconnection requires the membrane fusogen EFF-1 suggests that axonal fragments might directly activate fusogens or their regulators to promote reconnection.

Elevated $\mathrm{Ca}^{2+}$ or cAMP also stimulate regrowing PLM axon branching and synaptogenesis in the appropriate target region. The cues guiding the PLM ventral branching during development are not yet known; nevertheless, our findings suggest that such cues may persist in later stages. Although regrowing axons do not appear to be sensitive to these cues in the wild type, their sensitivity can be increased by elevated cAMP signaling. Loss of function in $c r h-1$ (CREB) did not affect developmental PLM branching but abrogated the effect of elevated CAMP on ventral branching of regrowing axons. CREB-dependent transcription may be specifically involved in the increased responsiveness of the regrowing axon to guidance cues.

The DLK-1 MAPKKK (mitogen-activated protein kinase kinase kinase) cascade is essential for regrowth of $C$. elegans touch neurons and motor neurons after injury (Hammarlund et al., 2009; Yan et al., 2009). Mammalian zipper protein kinase/dual leucine zipper kinase (DLK) has also been reported to be required for DRG neuron regrowth in culture, suggesting DLK kinases have a conserved role in regeneration (Itoh et al., 2009). We find that DLK-1 is not required for the $\mathrm{Ca}^{2+}$ transient in response to axotomy and that DLK-1 is required for regrowth even in conditions with elevated $\mathrm{Ca}^{2+}$ or cAMP signaling. These results are consistent with $\mathrm{Ca}^{2+}$ and cAMP signals either acting upstream of DLK-1, or via a target of DLK-1. One interpretation of these results is that DLK-1 pathway activity is simply permissive for any effects of $\mathrm{Ca}^{2+} / \mathrm{cAMP}$ signaling. However, we find that loss of function in the DLK-1 target CEBP-1 can be partly bypassed by elevated cAMP, although loss of function in CEBP-1 blocks regrowth to a similar extent to DLK-1 (Yan et al., 2009). These results suggest DLK-1 has other targets in addition to CEBP-1, some of which can be activated by elevated cAMP signaling.

\section{References}

Akerboom J, Rivera JD, Guilbe MM, Malave EC, Hernandez HH, Tian L, Hires SA, Marvin JS, Looger LL, Schreiter ER (2009) Crystal structures of the GCaMP calcium sensor reveal the mechanism of fluorescence signal change and aid rational design. J Biol Chem 284:6455-6464.

Appenzeller O, Palmer G (1972) The cyclic AMP (adenosine 3',5' phosphate) content of sciatic nerve: changes after nerve crush. Brain Res 42:521-524.

Baylis HA, Furuichi T, Yoshikawa F, Mikoshiba K, Sattelle DB (1999) Inositol 1,4,5-trisphosphate receptors are strongly expressed in the nervous 
system, pharynx, intestine, gonad and excretory cell of Caenorhabditis elegans and are encoded by a single gene (itr-1). J Mol Biol 294:467-476.

Berridge MJ (1993) Inositol trisphosphate and calcium signalling. Nature 361:315-325.

Bhatt DH, Otto SJ, Depoister B, Fetcho JR (2004) Cyclic AMP-induced repair of zebrafish spinal circuits. Science 305:254-258.

Bounoutas A, Zheng Q, Nonet ML, Chalfie M (2009) mec-15 encodes an F-box protein required for touch receptor neuron mechanosensation, synapse formation, and development. Genetics 183:607-617.

Bourgeois F, Ben-Yakar A (2008) Femtosecond laser nanoaxotomy properties and their effect on axonal recovery in C. elegans. Opt Express 16:5963.

Cai D, Deng K, Mellado W, Lee J, Ratan RR, Filbin MT (2002) Arginase I and polyamines act downstream from cyclic AMP in overcoming inhibition of axonal growth MAG and myelin in vitro. Neuron 35:711-719.

Carlsen RC (1982) Axonal transport of adenylate cyclase activity in normal and axotomized frog sciatic nerve. Brain Res 232:413-424.

Charlie NK, Thomure AM, Schade MA, Miller KG (2006) The Dunce cAMP phosphodiesterase PDE-4 negatively regulates $\mathrm{G}$ alpha(s)-dependent and G alpha(s)-independent cAMP pools in the Caenorhabditis elegans synaptic signaling network. Genetics 173:111-130.

Chen ZL, Yu WM, Strickland S (2007) Peripheral regeneration. Ann Rev Neurosci 30:209-233.

Dal Santo P, Logan MA, Chisholm AD, Jorgensen EM (1999) The inositol trisphosphate receptor regulates a 50 -second behavioral rhythm in $C$. elegans. Cell 98:757-767.

Filbin MT (2003) Myelin-associated inhibitors of axonal regeneration in the adult mammalian CNS. Nat Rev Neurosci 4:703-713.

Gabel CV, Antoine F, Chuang CF, Samuel AD, Chang C (2008) Distinct cellular and molecular mechanisms mediate initial axon development and adultstage axon regeneration in C. elegans. Development 135:1129-1136.

Guo SX, Bourgeois F, Chokshi T, Durr NJ, Hilliard MA, Chronis N, BenYakar A (2008) Femtosecond laser nanoaxotomy lab-on-a-chip for in vivo nerve regeneration studies. Nat Methods 5:531-533.

Hammarlund M, Nix P, Hauth L, Jorgensen EM, Bastiani M (2009) Axon regeneration requires a conserved MAP kinase pathway. Science 323:802-806.

Hoy RR, Bittner GD, Kennedy D (1967) Regeneration in crustacean motoneurons: evidence for axonal fusion. Science 156:251-252.

Itoh A, Horiuchi M, Bannerman P, Pleasure D, Itoh T (2009) Impaired regenerative response of primary sensory neurons in ZPK/DLK gene-trap mice. Biochem Biophys Res Commun 383:258-262.

Kamber D, Erez H, Spira ME (2009) Local calcium-dependent mechanisms determine whether a cut axonal end assembles a retarded endbulb or competent growth cone. Exp Neurol 219:112-125.

Kerr R, Lev-Ram V, Baird G, Vincent P, Tsien RY, Schafer WR (2000) Optical imaging of calcium transients in neurons and pharyngeal muscle of C. elegans. Neuron 26:583-594.

Kulbatski I, Cook DJ, Tator CH (2004) Calcium entry through L-type calcium channels is essential for neurite regeneration in cultured sympathetic neurons. J Neurotrauma 21:357-374.

Kwok TC, Ricker N, Fraser R, Chan AW, Burns A, Stanley EF, McCourt P, Cutler SR, Roy PJ (2006) A small-molecule screen in C. elegans yields a new calcium channel antagonist. Nature 441:91-95.

Lee RY, Lobel L, Hengartner M, Horvitz HR, Avery L (1997) Mutations in the alphal subunit of an L-type voltage-activated $\mathrm{Ca}^{2+}$ channel cause myotonia in Caenorhabditis elegans. EMBO J 16:6066-6076.

Lore AB, Hubbell JA, Bobb DS Jr, Ballinger ML, Loftin KL, Smith JW, Smyers ME, Garcia HD, Bittner GD (1999) Rapid induction of functional and morphological continuity between severed ends of mammalian or earthworm myelinated axons. J Neurosci 19:2442-2454.

Lu P, Yang H, Jones LL, Filbin MT, Tuszynski MH (2004) Combinatorial therapy with neurotrophins and cAMP promotes axonal regeneration beyond sites of spinal cord injury. J Neurosci 24:6402-6409.

Mohler WA, Shemer G, del Campo JJ, Valansi C, Opoku-Serebuoh E, Scranton V, Assaf N, White JG, Podbilewicz B (2002) The type I membrane protein EFF-1 is essential for developmental cell fusion. Dev Cell 2:355-362.
Nakai J, Ohkura M, Imoto K (2001) A high signal-to-noise Ca(2+) probe composed of a single green fluorescent protein. Nat Biotechnol 19:137-141.

Neumann S, Bradke F, Tessier-Lavigne M, Basbaum AI (2002) Regeneration of sensory axons within the injured spinal cord induced by intraganglionic cAMP elevation. Neuron 34:885-893.

Park K, Luo JM, Hisheh S, Harvey AR, Cui Q (2004) Cellular mechanisms associated with spontaneous and ciliary neurotrophic factor-cAMPinduced survival and axonal regeneration of adult retinal ganglion cells. J Neurosci 24:10806-10815.

Pichichero M, Beer B, Clody DE (1973) Effects of dibutyryl cyclic AMP on restoration of function of damaged sciatic nerve in rats. Science 182:724-725.

Pujol N, Cypowyj S, Ziegler K, Millet A, Astrain A, Goncharov A, Jin Y, Chisholm AD, Ewbank JJ (2008) Distinct innate immune responses to infection and wounding in the C. elegans epidermis. Curr Biol 18:481-489.

Raivich G, Bohatschek M, Da Costa C, Iwata O, Galiano M, Hristova M, Nateri AS, Makwana M, Riera-Sans L, Wolfer DP, Lipp HP, Aguzzi A, Wagner EF, Behrens A (2004) The AP-1 transcription factor c-Jun is required for efficient axonal regeneration. Neuron 43:57-67.

Rolls MM, Hall DH, Victor M, Stelzer EH, Rapoport TA (2002) Targeting of rough endoplasmic reticulum membrane proteins and ribosomes in invertebrate neurons. Mol Biol Cell 13:1778-1791.

Sands WA, Palmer TM (2008) Regulating gene transcription in response to cyclic AMP elevation. Cell Signal 20:460-466.

Schade MA, Reynolds NK, Dollins CM, Miller KG (2005) Mutations that rescue the paralysis of Caenorhabditis elegans ric- 8 (synembryn) mutants activate the $\mathrm{G}$ alpha(s) pathway and define a third major branch of the synaptic signaling network. Genetics 169:631-649.

Spencer T, Filbin MT (2004) A role for cAMP in regeneration of the adult mammalian CNS. J Anat 204:49-55.

Suzuki H, Kerr R, Bianchi L, Frokjaer-Jensen C, Slone D, Xue J, Gerstbrein B, Driscoll M, Schafer WR (2003) In vivo imaging of C. elegans mechanosensory neurons demonstrates a specific role for the MEC-4 channel in the process of gentle touch sensation. Neuron 39:1005-1017.

Tsien RY (1981) A non-disruptive technique for loading calcium buffers and indicators into cells. Nature 290:527-528.

Tsujino H, Kondo E, Fukuoka T, Dai Y, Tokunaga A, Miki K, Yonenobu K, Ochi T, Noguchi K (2000) Activating transcription factor 3 (ATF3) induction by axotomy in sensory and motoneurons: A novel neuronal marker of nerve injury. Mol Cell Neurosci 15:170-182.

Walker DS, Gower NJ, Ly S, Bradley GL, Baylis HA (2002) Regulated disruption of inositol 1,4,5-trisphosphate signaling in Caenorhabditis elegans reveals new functions in feeding and embryogenesis. Mol Biol Cell 13:1329-1337.

Wolf JA, Stys PK, Lusardi T, Meaney D, Smith DH (2001) Traumatic axonal injury induces calcium influx modulated by tetrodotoxin-sensitive sodium channels. J Neurosci 21:1923-1930.

Wu Z, Ghosh-Roy A, Yanik MF, Zhang JZ, Jin Y, Chisholm AD (2007) Caenorhabditis elegans neuronal regeneration is influenced by life stage, ephrin signaling, and synaptic branching. Proc Natl Acad Sci U S A 104:15132-15137.

Xu K, Tavernarakis N, Driscoll M (2001) Necrotic cell death in C. elegans requires the function of calreticulin and regulators of $\mathrm{Ca}(2+)$ release from the endoplasmic reticulum. Neuron 31:957-971.

Yan D, Wu Z, Chisholm AD, Jin Y (2009) The DLK-1 kinase promotes mRNA stability and local translation in C. elegans synapses and axon regeneration. Cell 138:1005-1018.

Yanik MF, Cinar H, Cinar HN, Chisholm AD, Jin Y, Ben-Yakar A (2004) Neurosurgery: functional regeneration after laser axotomy. Nature 432:822.

Yiu G, He Z (2006) Glial inhibition of CNS axon regeneration. Nat Rev Neurosci 7:617-627.

Ziv NE, Spira ME (1993) Spatiotemporal distribution of Ca2+ following axotomy and throughout the recovery process of cultured Aplysia neurons. Eur J Neurosci 5:657-668.

Ziv NE, Spira ME (1995) Axotomy induces a transient and localized elevation of the free intracellular calcium concentration to the millimolar range. J Neurophysiol 74:2625-2637. 